\title{
Constructing an entangled state in the Heisenberg picture for inflationary cosmology
}

\author{
Abasalt Rostami* \\ Department of Physics, Sharif University of Technology, Tehran, Iran \\ and School of Physics, Institute for Research in Fundamental Sciences (IPM), \\ P. O. Box 19395-5531, Tehran, Iran \\ Javad T. Firouzjaee \\ School of Astronomy, Institute for Research in Fundamental Sciences (IPM), \\ P. O. Box 19395-5531, Tehran, Iran
}

(Received 3 December 2017; published 1 March 2018)

\begin{abstract}
The effects of the entanglement of the inflaton field which initially entangled with those of another field on observables like power spectrum are known in the context of the Schrödinger field theory. To clarify this effect in the Heisenberg picture, there were some attempts to construct the initial entangled state by making use of an entangled transformation (like Bogoliubov transformation) between the Bunch-Davies vacuums and squeezed states. We study the role of the time-dependent entangled transformation in the Schrödinger field theory. We derive the relation between two vacuum states which their mode functions are transformed by the entangled transformation in Schrödinger picture. We discuss that the time-dependency of the entanglement parameter is inevitable and only in the first order of the entanglement parameter perturbation this time-dependency vanishes. We study the entangled transformation in the Heisenberg picture in term of the entangled parameter which appears in the entangled state in Schrödinger picture.
\end{abstract}

DOI: 10.1103/PhysRevD.97.063501

\section{INTRODUCTION}

The main experimental observation of the early universe, cosmic microwave background (CMB), have become very accurate and have allowed us to deduce the details of the even earlier phase of the cosmos [1]. It is known that the temperature fluctuation in the $\mathrm{CMB}$ are originated from the early quantum fluctuation which becomes classical as the universe expand. Therefore, studying the quantum nature of the inflationary background can lead to better understanding of the observables like power spectrum and bispectrum which can have quantum correction.

An important aspect of the quantum physics is the entanglement which has been one of the fascinating features predicted by quantum mechanics since Einstein-PodolskyRosen (EPR) [2]. Although more attention has been paid to how to make use of quantum entanglement of EPR pairs in quantum cryptography and quantum information [3], the

\footnotetext{
*aba-rostami@ipm.ir

j.taghizadeh.f@ipm.ir
}

Published by the American Physical Society under the terms of the Creative Commons Attribution 4.0 International license. Further distribution of this work must maintain attribution to the author(s) and the published article's title, journal citation, and DOI. Funded by SCOAP. entanglement entropy plays an important role in studying the black hole information problem and holography which we can count of the degrees of entanglement of a quantum system. In the cosmology, Maldacena [4] developed an explicit method to calculate the entanglement entropy in a quantum field theory in the Bunch-Davies vacuum of the de Sitter space and showed that there can be the quantum entanglement between two causally disconnected open charts in de Sitter space and in this way more ideas presented to detect these quantum effects [5]. Recently, different perspectives for the quantum entanglement proposed to study the nature of the dark energy (cosmological constant). In this sense, one can make the cosmological constant as a remnant of the quantum decoherence process for the dark energy field from other d.o.f. in the environment [6]. In this way, the dynamical dark energy can be generated as a byproduct of quantum entanglement between different cosmological epochs [7] and the dark energy is interpreted as a signature of entanglement from the quantum decoherence [8]. This view opens different questions about the information evolution and its preservation in the Friedmann-Lemaitre-Robertson-Walker (FLRW) universe [9]. Along the way, one can study the quantum entanglement in the Schrödinger field theory which the operators are time independent. The Schrödinger picture is the natural framework to study the entanglement between the fields [10]. The 
Schrödinger representation is also best suited to discuss the decoherence of the cosmological perturbations and the quantum to classical transition in inflationary models [11] and one can develop in-in formalism in the Schrödinger picture for curvature perturbations [12] to study N-point function in the observation. In the way, many works have been done to study the entanglement effect and the quantum to classical transition in the inflationary models [13].

On the other side, apart from this fact that the Heisenberg picture field theory is more common and easier to study the inflationary models (for example the calculation of the Power spectrum and non-Gaussianity in the Schrödinger picture needs the heavy calculation on the functional space), this picture has better description for particle representation of each state with explicit presentation of annihilation and creation process. Moreover, constructing the entangled states using the entanglement transformation for fermions is easier in the Heisenberg picture than the Schrödinger picture [14]. There is an instruction to built the entangled state in the Heisenberg picture.

Recently, in [15] an initially entangled state between two free massive scalar fields in de Sitter space was studied and the entangled state using a Bogoliubov transformation between the Bunch-Davies vacuum and a four-mode squeezed state was constructed. In this paper, we show that though Kanno [15] presented a novel way to study the entangled state in the Heisenberg picture, her calculation and analysis to introduce the entanglement was not correct in some parts. We follow her way to study the role of the time-dependent entangled transformation in the Schrödinger field theory. We derive the relation between two vacuum states which their mode functions are transformed by the entangled transformation and also Bogoliubov transformation in Schrödinger picture. We calculate the entangled parameter in Schrödinger picture in term of the time-dependent entangled transformation in the Heisenberg picture, and the inflation power spectrum will be calculated in term of the time-dependent entangled parameter in Heisenberg picture. It is discussed that the time-dependency of the entanglement parameter is inevitable and only in the first order of the entanglement parameter perturbation this time-dependency vanishes. We will discuss the limitation that the current definition of the entanglement.

The paper is organized as follows. First, we review the quantization of a monofield for FLRW background in Sec. II. Section III is devoted to introduce and use the Bogoliubov transformation to get the vacuum state in the Schrödinger picture. In Sec. IV we introduce the AlbrechtBolis-Holman (ABH) [10] entangled state in the Schrödinger picture of the inflationary cosmology. Next, after discussing the relation between the $\mathrm{ABH}$ entangled state and the entangled stated which built of the entangled transformation, the inflationary power spectrum will be calculated in Sec. V. We end with a discussion in Sec. VI.

\section{A SHORT REVIEW ON MONOFIELD QUANTIZATION}

The main goal of this section is to study a free scalar field in time-dependent gravitational backgrounds. As a first step, we shall consider a free scalar field in a homogeneous and isotropic universe and then try to quantize it. In this way, we follow Mukhanov and Winitzki book notation [16].

A minimally coupled real scalar field (x) in a curved spacetime is described by the action

$$
S[\phi]=\int d^{4} x \sqrt{-g}\left(\frac{1}{2} g^{\alpha \beta} \partial_{\alpha} \phi \partial_{\beta} \phi-\frac{1}{2} m^{2} \phi^{2}\right) .
$$

Now, we consider an important class of metrics which is used for the homogeneous and isotropic space-time namely FLRW. This is characterized by the following metric

$$
d s^{2}=-d t^{2}+a^{2}(t) d \vec{x}^{2} .
$$

where $a(t)$ is the scale factor. A flat FRW space-time is a conformally flat space-time. If one replaces the coordinate $t$ by the conformal time $\eta$, the metric would be transformed into a conformally flat form

$$
\eta(t) \equiv \int_{t_{0}}^{t} \frac{d t}{a(t)} .
$$

By this transformation the action (1) is written as

$$
S[\phi]=\frac{1}{2} \int d^{3} x d \eta a^{2}\left(\phi^{2}-(\nabla \phi)^{2}-m^{2} a^{2} \phi^{2}\right) .
$$

where a prime denotes the derivative with respect to the conformal time $\eta$. This action gives the following equation of motion for our scalar field

$$
\phi^{\prime \prime 2}-\nabla^{2} \phi+2 \frac{a^{\prime}}{a} \phi^{\prime}+m^{2} a^{2} \phi=0 .
$$

Although this equation seems a bit different from the equation of motion which one derives for a scalar field in flat space-time but by using of the field redefinition $\tilde{\phi}=a \phi$, we could make it more similar to the equation of motion a scalar field in flat background. In fact with this redefinition the action for $\tilde{\phi}$ is

$$
S[\tilde{\phi}]=\frac{1}{2} \int d^{3} x d \eta\left(\tilde{\phi}^{\prime 2}-(\nabla \tilde{\phi})^{2}-m^{2}(\eta) \tilde{\phi}^{2}\right)
$$

where, we have defined

$$
m^{2}(\eta)=m^{2} a^{2}-\frac{a^{\prime \prime}}{a} .
$$

Now, the equation of motion for the new field is 


$$
\tilde{\phi}^{\prime \prime}-\nabla^{2} \tilde{\phi}+m^{2}(\eta) \tilde{\phi}=0
$$

this equation is like what we had in flat space-time for a free field with this difference that here the mass term is time dependent. All information about the influence of the gravitational field on $\tilde{\phi}$ is encapsulated in the timedependent mass $m(\eta)$. This would lead to this fact that the energy of the field $m(\eta)$ is generally not conserved. In quantum physics language, this leads to the possibility of particle creation which the energy for new particles is supplied by the gravitational field. If we expand the field $\tilde{\phi}$ in Fourier modes, we have

$$
\tilde{\phi}(\eta, x)=\int \frac{d^{3} k}{(2 \pi)^{3}} \tilde{\phi}_{k}(\eta) e^{i k \cdot x} .
$$

Thus, reach to the decoupled equations of motion for the modes $\tilde{\phi}_{k}$

$$
\tilde{\phi}_{k}^{\prime \prime 2}+\omega_{k}^{2}(\eta) \tilde{\phi}_{k}=0
$$

where we have defined

$$
\omega_{k}^{2}(\eta) \equiv k^{2}+m^{2}(\eta)
$$

This equation has two linearly independent solutions for each mode which these two solutions span the two-dimensional space of all solutions. If $v_{k}(\eta)$ is a complex solution then $v_{k}^{*}(\eta)$ would be another independent solution. Any two linearly independent solutions have to have a nonvanishing Wronskian. One can easily show the Wronskian of any two independent solutions of (10) is constant in conformal time. We choose this constant as following

$$
W\left(v_{k}, v_{k}^{*}\right)=v_{k}^{\prime} v_{k}^{*}-v_{k} v_{k}^{\prime *}=2 i .
$$

The general solution $\tilde{\phi}_{k}$ can be expressed as a linear combination of $v_{k}^{*}(\eta)$ and $v_{k}(\eta)$ as

$$
\tilde{\phi}_{k}(\eta)=\frac{1}{\sqrt{2}}\left[a_{k} v_{k}^{*}(\eta)+a_{-k}^{*} v_{k}(\eta)\right]
$$

where the $v_{k}^{*}(\eta)$ and $v_{k}(\eta)$ are called mode functions. Now, by replacing (13) in (9), we obtain $\tilde{\phi}(\eta, x)$

$\tilde{\phi}(\eta, x)=\int \frac{d^{3} k}{(2 \pi)^{\frac{3}{2}}} \frac{1}{\sqrt{2}}\left[a_{k} v_{k}^{*}(\eta) e^{i k \cdot x}+a_{k}^{*} v_{k}(\eta) e^{-i k \cdot x}\right]$.

Note that, the reality condition for $\phi(\eta, x)$ entails $\tilde{\phi}_{k}=\tilde{\phi}_{-k}^{*}$. To obtain a quantum picture of such theory, one has to consider $a_{k}$ and its conjugate $a_{k}^{\dagger}$ as operators to construct the Hilbert space. Thus, at first we should write down $a_{k}$ as a combination of $\tilde{\phi}_{k}$ and its conjugate momentum. Using Eqs. (13) and (12) we find $a_{k}$

$$
a_{k}=\frac{1}{i \sqrt{2}}\left(v_{k}^{\prime}(\eta) \tilde{\phi}_{k}(\eta)-\tilde{\phi}_{k}^{\prime}(\eta) v_{k}(\eta)\right)
$$

and its conjugate

$$
a_{k}^{*}=-\frac{1}{i \sqrt{2}}\left(v_{k}^{\prime *}(\eta) \tilde{\phi}_{-k}(\eta)-\tilde{\phi}_{-k}^{\prime}(\eta) v_{k}^{*}(\eta)\right) .
$$

Since the conjugate momentum of $\tilde{\phi}$ is

$$
\tilde{\Pi}(\eta, x)=\tilde{\phi}^{\prime}(\eta, x)
$$

we have

$$
a_{k}=\frac{1}{i \sqrt{2}}\left(v_{k}^{\prime}(\eta) \tilde{\phi}_{k}(\eta)-\tilde{\Pi}_{k}(\eta) v_{k}(\eta)\right)
$$

and

$$
a_{k}^{*}=-\frac{1}{i \sqrt{2}}\left(v_{k}^{*}(\eta) \tilde{\phi}_{-k}(\eta)-\tilde{\Pi}_{-k}(\eta) v_{k}^{*}(\eta)\right)
$$

Now, to reach a quantum picture, it is enough to define the usual following commutator

$$
[\tilde{\phi}(\eta, x), \tilde{\Pi}(\eta, y)]=i \delta(x-y) .
$$

This gives a representation for momentum as

$$
\tilde{\Pi}_{k}(\eta)=-i(2 \pi)^{3} \frac{\delta}{\delta \tilde{\phi}_{-k}(\eta)} .
$$

The above equation leads to the usual commutation relation for creation and annihilation operators

$$
\left[a_{k}, a_{p}^{\dagger}\right]=(2 \pi)^{3} \delta(k-p) .
$$

Once the operators $a_{k}$ and $a_{k}^{\dagger}$ are determined, the vacuum state $|0\rangle$ is defined as the eigenstate of all annihilation operators $a_{k}$ with eigenvalue 0, i.e. $a_{k}|0\rangle=0$ for all $\mathrm{k}$. An excited state $\left|n_{k_{1}}, m_{k_{2}}, \ldots\right\rangle$ with the occupation numbers $m, n, \ldots$ in the modes $\tilde{\phi}_{k_{1}}, \tilde{\phi}_{k_{2}}, \ldots$ is then given by

$\left|n_{k_{1}}, m_{k_{2}}, \cdots\right\rangle=\frac{1}{\sqrt{n ! m ! \ldots}}\left[\left(a_{k_{1}}^{\dagger}\right)^{n}\left({a_{k_{2}}}^{\dagger}\right)^{m} \cdots\right]\left|0_{k_{1}}, 0_{k_{2}}, \cdots\right\rangle$

The space spanned by these vectors is the physical Hilbert space and therefore every quantum state can be expressed as below

$$
|\Psi\rangle=\sum_{n, m, \ldots} C_{n, m, \ldots}\left|n_{k_{1}}, m_{k_{2}}, \cdots\right\rangle,
$$


where $\left|C_{n, m, \ldots}\right|^{2}$ defines the probability of finding system in the corresponding excited state. Note that the inner product for a such Hilbert space is simply defined just by using Eq. (22) and this request that $\langle 0 \mid 0\rangle=1$.

It is interesting to know what is Schrödinger picture of vacuum state. After determining this state one could use (19) and (22) to derive exited states in this picture. To derive the explicit form of vacuum, we use this fact that for each mode $k$

$$
\left\langle\tilde{\phi}_{k}\left|a_{k}\right| 0\right\rangle=0,
$$

where $\left|\tilde{\phi}_{k}\right\rangle$ is eigenstate of $\tilde{\phi}_{k}$. Now using (18), we will get a simple functional differential equation (i continuous limit) with the following solution

$\Psi[\tilde{\phi}]=\prod_{k}\left\langle\tilde{\phi}_{k} \mid 0\right\rangle=N(\eta) \exp \left[\frac{i}{2} \int \frac{d^{3} k}{(2 \pi)^{3}} \frac{v_{k}^{\prime}}{v_{k}}(\eta) \tilde{\phi}_{k} \tilde{\phi}_{-k}\right]$

where $N(\eta)$ is the normalization factor and can be calculated by using of

$$
\int D \tilde{\phi} \Psi[\tilde{\phi}] \Psi^{*}[\tilde{\phi}]=1
$$

for the measure $D \tilde{\phi}=d \tilde{\phi}_{k_{1}} d \tilde{\phi}_{-k_{1}} d \tilde{\phi}_{k_{2}} d \tilde{\phi}_{-k_{2}} \ldots$ It is easy to show that apart of a constant for a given $k$-mode, we have $N_{k} \sim \frac{1}{\left|v_{k}(\eta)\right|}$. In the mathematical point of view, the wave function $\Psi[\tilde{\phi}]$ is defined on functional space of an infinite dimensional manifold. People rarely use this picture of quantum field theory because occupation number of each state is not manifest in this picture. However, some kind of calculations is easier than Heisenberg picture. To end up this section for future advantages, we derive wave function of the main field i.e. $\phi$. One can check out the annihilation and creation operators for this field would be

$a_{k}=\frac{1}{i \sqrt{2}}\left[\left(a v_{k}^{\prime}(\eta)-a^{\prime} v_{k}(\eta)\right) \phi_{k}(\eta)+i(2 \pi)^{3} \frac{v_{k}}{a}(\eta) \frac{\delta}{\delta \phi_{-k}}\right]$

and

$$
\begin{aligned}
a_{k}^{\dagger}= & -\frac{1}{i \sqrt{2}}\left[\left(a v_{k}^{* \prime}(\eta)-a^{\prime} v_{k}^{*}(\eta)\right) \phi_{-k}(\eta)\right. \\
& \left.+i(2 \pi)^{3} \frac{v_{k}^{*}}{a}(\eta) \frac{\delta}{\delta \phi_{k}}\right] .
\end{aligned}
$$

Now, we could find the wave function as

$$
\begin{aligned}
\Psi[\phi] & =\prod_{k}\left\langle\phi_{k} \mid 0\right\rangle \\
& =N(\eta) \exp \left[\frac{i}{2} \int \frac{d^{3} k}{(2 \pi)^{3}}\left(\frac{v_{k}^{\prime}}{v_{k}}(\eta)-\frac{a^{\prime}}{a}(\eta)\right) \phi_{k} \phi_{-k}\right]
\end{aligned}
$$

and we see that the difference is just $\frac{a^{\prime}}{a}$ in the exponent which called Hubble factor. Note that one could use the Schrödinger equation in functional space to reach this wave function. We shall do it for two entangled scalar field later on. We will end this section with some comments about the Hilbert space in Schrödinger picture (for our case in this paper). There are two ways to construct a Hilbert state in Schrödinger picture. The first (and the natural) way is using the Schrödinger equation and finding its independent solutions. These solutions live in a $L^{2}$ function space for a theory with only finite d.o.f. (classical quantum theory). In the case of infinite d.o.f. i.e. quantum field theory, the Hamiltonian is a functional of given fields and Schrödinger equation is a functional differential equation. Therefore, every solution of this equation is a functional. These functionals construct the corresponding Hilbert space with the following inner product

$$
(\Phi, \Psi)=\int D \tilde{\phi} \Psi[\tilde{\phi}] \Phi^{*}[\tilde{\phi}]
$$

Another (sometimes easier) way to construct the Hilbert space in Schrödinger picture is using the representation theory. Having established the Hilbert space in Heisenberg picture for a field theory (which is known as an abstract Fock space), one can find its functional representation. To do this we have to find the eigenvalues of the field operators for each mode in the Fourier space i.e. $\hat{\tilde{\phi}}_{k} \mathrm{~s}$ [which can be expressed in term of annihilation and creation operators. See Eq. (13)]

$$
\tilde{\tilde{\phi}}_{k}\left|\tilde{\phi}_{k}\right\rangle=\tilde{\phi}_{k}\left|\tilde{\phi}_{k}\right\rangle .
$$

Using these vectors one would be able to define the wave functional basis as following

$$
\Psi_{n, m, \cdots}[\tilde{\phi}]=\left(\prod_{k}\left\langle\tilde{\phi}_{k}\right)\left|n_{k_{1}}, m_{k_{2}}, \cdots\right\rangle .\right.
$$

Under the above inner product these (independent) functionals define the physical Hilbert space. Now every vector $\Psi[\phi]$ [corresponding to $|\Psi\rangle$ in Eq. (24)] in this space can be expressed as

$$
\Psi[\phi]=\left(\prod_{k}\left\langle\tilde{\phi}_{k}\right)|\Psi\rangle=\sum_{n, m, \ldots} C_{n, m, \ldots} \Psi_{n, m, \ldots}[\tilde{\phi}]\right.
$$




\section{BOGOLIUBOV TRANSFORMATION}

Consider two sets of isotropic mode functions $u_{k}(\eta)$ and $v_{k}(\eta)$ are chosen. Since $v_{k}$ and $u_{k}^{*}$ are a basis, the function $u_{k}$ must be a linear combination of $v_{k}$ and $u_{k}^{*}$,

$$
u_{k}(\eta)=\alpha_{k} v_{k}(\eta)-\beta_{k}^{*} v_{k}^{*}(\eta)
$$

with time-independent complex coefficients $\alpha_{k}$ and $\beta_{k}$. If both sets $v_{k}$ and $u_{k}$ are normalized by (12), it follows that the coefficients $\alpha_{k}$ and $\beta_{k}$ satisfy

$$
\left|\alpha_{k}\right|^{2}-\left|\beta_{k}\right|^{2}=1
$$

Using the mode functions $u_{k}$ instead of $v_{k}$, one obtains an alternative mode expansion which defines another set $\tilde{a}_{k}$ and $\tilde{a}_{k}^{\dagger}$ of creation and annihilation operators

$\tilde{\phi}(\eta, x)=\int \frac{d^{3} k}{(2 \pi)^{\frac{3}{2}}} \frac{1}{\sqrt{2}}\left[\tilde{a}_{k} u_{k}^{*}(\eta) e^{i k . x}+\tilde{a}_{k}^{*} u_{k}(\eta) e^{-i k \cdot x}\right]$,

in which we have defined

$$
\tilde{a}_{k}=\alpha_{k} a_{k}+\beta_{k}^{*} a_{-k}^{*}
$$

Now one can refer a new vacuum state $|\tilde{0}\rangle$ respect to $\tilde{a}_{k}$ which annihilated by this operator and then by expanding the new vacuum in term of previous basis (23) to reach

$$
|\tilde{0}\rangle=\Pi_{k} \frac{1}{\sqrt{\left|\alpha_{k}\right|}} \exp \left(-\frac{\lambda_{k}}{2} a_{k}^{\dagger} a_{-k}^{\dagger}\right)|0\rangle
$$

where

$$
\lambda_{k}=\frac{\beta_{k}^{*}}{\alpha_{k}} .
$$

This is an infinite series which can be express explicitly for each mode as

$$
\left|0_{k}, 0_{-k}\right\rangle=\frac{1}{\left|\alpha_{k}\right|} \sum_{n=0}^{\infty}\left(-\frac{\beta_{k}^{*}}{\alpha_{k}}\right)^{n}\left|n_{k}, n_{-k}\right\rangle .
$$

The $\alpha_{k}$ and $\beta_{k}$ constants are called Bogoliubov constants. It is interesting to know the closed exact form of the
Bogoliubov transformation in the Schrödinger picture [17]. To do it, we need to have the new vacuum in the Schrödinger picture. It is obvious that

$$
\prod_{k}\left\langle\tilde{\phi}_{k} \mid \tilde{0}\right\rangle=\tilde{N} \exp \left[\frac{i}{2} \int \frac{d^{3} k}{(2 \pi)^{3}} \frac{u_{k}^{\prime}}{u_{k}}(\eta) \tilde{\phi}_{k} \tilde{\phi}_{-k}\right]
$$

because the difference between two vacuums is in mode functions and it is enough to replace $v_{k}$ with $u_{k}$. Using this fact that $\left|\lambda_{k} \frac{v_{k}^{*}}{v_{k}}\right|<1$ and expanding $\frac{u_{k}^{\prime}}{u_{k}}$ in term of $\frac{v_{k}^{\prime}}{v_{k}}$, one obtains

$$
\begin{aligned}
\prod_{k}\left\langle\tilde{\phi}_{k} \mid \tilde{0}\right\rangle= & N \exp \left[\frac{i}{2} \int \frac{d^{3} k}{(2 \pi)^{3}} \frac{v_{k}^{\prime}}{v_{k}}(\eta) \tilde{\phi}_{k} \tilde{\phi}_{-k}\right] \\
& \times\left(\prod_{k} \frac{1}{\left|\alpha_{k}\right|} \frac{1}{\left.\mid 1-\lambda_{k} \frac{v_{k}^{*} \mid}{v_{k}}\right)}\right. \\
& \times \exp \left(-\sum_{n=0}^{\infty} \int \frac{d^{3} k}{(2 \pi)^{3}} \lambda_{k}^{n+1} \frac{1}{v_{k}^{2}}\left(\frac{v_{k}^{*}}{v_{k}}\right)^{n} \tilde{\phi}_{k} \tilde{\phi}_{-k}\right)
\end{aligned}
$$

This is a magic relation because not only expresses the new vacuum in a closed form of previous basis but also could be used to derive other exited states in the Schrödinger picture. One can expand two last terms in Eq. (43) and compare $\lambda_{k}^{n}$ terms with their counterpart in (41) to find all exited states. Before ending this section, it is worth to mention a very important point that all information about kind of a state is hidden in mode functions. For example, by looking at (42) one cannot observe any entanglement in this state. Therefore, to see this entanglement between excited states, we have to study mode functions of one observer and compare it with another observer.

\section{ABH STATE}

In 2014, ABH [10] introduced an interesting state which describes entanglement between two noninteracting scalar fields in FLRW background. They applied it in Schrödinger picture and use it as an ansats to solve Schrödinger equation. In this section, we have a short review of their derivation for more details see [10]. Suppose two fields $\phi$ and $\chi$ coupled to gravity with no any other interactions,

$$
S=\frac{1}{2} \int d^{4} x a^{4}(\eta)\left[\frac{1}{a^{2}(\eta)}\left(\Phi^{\prime}(\eta, \vec{x})^{2}-(\nabla \Phi(\eta, \vec{x}))^{2}\right)-m_{\Phi}^{2} \Phi(\eta, \vec{x})^{2}+\frac{1}{a^{2}(\eta)}\left(\chi^{\prime}(\eta, \vec{x})^{2}-(\nabla \chi(\eta, \vec{x}))^{2}\right)-m_{\chi}^{2} \chi(\eta, \vec{x})^{2}\right],
$$

To be able to make a functional Schrödinger equation for a such action one needs the Hamiltonian in terms of conjugate momenta; 
$H=\int d^{3} x\left[\frac{\pi_{\Phi}^{2}}{2 a^{2}(\eta)}+\frac{1}{2} a^{2}(\eta)\left((\nabla \Phi(\eta, \vec{x}))^{2}+a^{2}(\eta) m_{\Phi}^{2} \Phi(\eta, \vec{x})^{2}\right)+\frac{\pi_{\chi}^{2}}{2 a^{2}(\eta)}+\frac{1}{2} a^{2}(\eta)\left((\nabla \chi(\eta, \vec{x}))^{2}+a^{2}(\eta) m_{\Phi}^{2} \chi(\eta, \vec{x})^{2}\right)\right]$

where $\pi_{\phi}$ and $\pi_{\chi}$ are the canonically conjugate momenta for $\phi$ and $\chi$. Like the previous section, it would be more appropriate to go to Fourier space as following for $\phi$

$\Phi(\eta, \vec{x})=\sum_{\vec{k}} \frac{\phi_{\vec{k}}}{\sqrt{V}} e^{-i \vec{k} \cdot \vec{x}}, \quad \pi_{\Phi}(\eta, \vec{x})=\sum_{\vec{k}} \frac{\pi_{\Phi, \vec{k}}}{\sqrt{V}} e^{-i \vec{k} \cdot \vec{x}}$

and with a similar transformation for $\chi$. It is easy to see with this decomposition, the Hamiltonian has the following form

$$
\begin{aligned}
H & =H_{\Phi}+H_{\chi} \quad H_{\Phi}=\sum_{\vec{k}} H_{\Phi, \vec{k}}, \\
H_{\Phi, \vec{k}} & =\frac{\pi_{\Phi, \vec{k}} \pi_{\Phi,-\vec{k}}}{2 a^{2}(\eta)}+\frac{1}{2} a^{2}(\eta)\left(k^{2}+m_{\Phi}^{2} a^{2}(\eta)\right) \phi_{\vec{k}} \phi_{-\vec{k}} \\
H_{\chi} & =\sum_{\vec{k}} H_{\chi, \vec{k}}, \\
H_{\chi, \vec{k}} & =\frac{\pi_{\chi, \vec{k}} \pi_{\chi,-\vec{k}}}{2 a^{2}(\eta)}+\frac{1}{2} a^{2}(\eta)\left(k^{2}+m_{\chi}^{2} a^{2}(\eta)\right) \chi_{\vec{k}} \chi_{-\vec{k}} .
\end{aligned}
$$

Now, in this level one can use the separation of variables method to solve Schrödinger equation for each $k$-mode

$$
i \partial_{\eta} \psi_{\vec{k}}\left[\phi_{\vec{k}}, \chi_{\vec{k}} ; \eta\right]=\left(H_{\Phi, \vec{k}}+H_{\chi, \vec{k}}\right) \psi_{\vec{k}}\left[\phi_{\vec{k}}, \chi_{\vec{k}} ; \eta\right]
$$

but such solution is only able to give an entangled state between exited states of one kind of field not entanglement between different fields. To make a entangled state between different fields, we use the following ansats

$$
\begin{aligned}
\psi_{\vec{k}}\left[\phi_{\vec{k}}, \chi_{\vec{k}} ; \eta\right]= & N_{k}(\eta) \exp \left[-\frac{1}{2}\left(A_{k}(\eta) \phi_{\vec{k}} \phi_{-\vec{k}}+B_{k}(\eta) \chi_{\vec{k}} \chi_{-\vec{k}}\right.\right. \\
& \left.\left.+C_{k}(\eta)\left(\phi_{\vec{k}} \chi_{-\vec{k}}+\chi_{\vec{k}} \phi_{-\vec{k}}\right)\right)\right] .
\end{aligned}
$$

where $C_{k}$ plays the role of entanglement between two fields. Inserting (48) into (47) and then matching the powers of the field modes gives us the following equations

$$
\begin{aligned}
i \frac{N_{k}^{\prime}}{N_{k}} & =\frac{\left(A_{k}+B_{k}\right)}{2 a^{2}(\eta)} \\
i A_{k}^{\prime} & =\frac{A_{k}^{2}+C_{k}^{2}}{a^{2}(\eta)}-\Omega_{\Phi, k}^{2} a^{2}(\eta), \quad \Omega_{\Phi, k}^{2} \equiv k^{2}+m_{\Phi}^{2} a^{2}(\eta) \\
i B_{k}^{\prime} & =\frac{B_{k}^{2}+C_{k}^{2}}{a^{2}(\eta)}-\Omega_{\chi, k}^{2} a^{2}(\eta), \quad \Omega_{\chi, k}^{2} \equiv k^{2}+m_{\chi}^{2} a^{2}(\eta) \\
i \frac{C_{k}^{\prime}}{C_{k}} & =\frac{\left(A_{k}+B_{k}\right)}{a^{2}(\eta)} .
\end{aligned}
$$

One could reach a more suitable form of these equations using the following definitions

$$
\begin{aligned}
& i A_{k}(\eta) \equiv a^{2}(\eta)\left(\frac{f_{k}^{\prime}(\eta)}{f_{k}(\eta)}-\frac{a^{\prime}(\eta)}{a(\eta)}\right), \\
& i B_{k}(\eta) \equiv a^{2}(\eta)\left(\frac{g_{k}^{\prime}(\eta)}{g_{k}(\eta)}-\frac{a^{\prime}(\eta)}{a(\eta)}\right)
\end{aligned}
$$

Then the resulting differential equation under these definitions would be

$$
\begin{gathered}
f_{k}^{\prime \prime}+\left(\Omega_{\Phi, k}^{2}-\frac{a^{\prime \prime}(\eta)}{a(\eta)}\right) f_{k}=\frac{C_{k}(\eta)^{2}}{a^{4}(\eta)} f_{k} \\
g_{k}^{\prime \prime}+\left(\Omega_{\chi, k}^{2}-\frac{a^{\prime \prime}(\eta)}{a(\eta)}\right) g_{k}=\frac{C_{k}(\eta)^{2}}{a^{4}(\eta)} g_{k}
\end{gathered}
$$

and furthermore $C_{k}(\eta)$ would be determined by

$$
\frac{C_{k}(\eta)}{a^{2}(\eta)}=\frac{c_{k}}{f_{k}(\eta) g_{k}(\eta)}
$$

which we call $c_{k}$ the entanglement parameter and if this parameter vanishes then we regain (26). Note that this parameter is constant of the integral and only depends on $k$. Here it is enough to solve coupled differential equations in (51) with given initial conditions. ABH showed that these equations have a perturbative solution of even orders of entanglement parameter $c_{k}^{2 n}$ for $f_{k}$ and odd orders $c_{k}^{2 n+1}$ for $C_{k}$. In fact if $v_{k}$ and $u_{k}$ be solutions of Eq. (52) for $f_{k}$ and $g_{k}$ respectively when $c_{k}=0$, then they found

$$
\begin{gathered}
f_{k}(\eta)=v_{k}(\eta)\left(1+c_{k}^{2} \mathcal{F}_{k}(\eta)+\cdots\right), \\
g_{k}(\eta)=u_{k}(\eta)\left(1+c_{k}^{2} \mathcal{G}_{k}(\eta)+\cdots\right)
\end{gathered}
$$

and 


$$
C_{k}=\frac{c_{k}}{u_{k}(\eta) v_{k}(\eta)}-\frac{c_{k}^{3}}{u_{k}(\eta) v_{k}(\eta)}\left(\mathcal{F}_{k}(\eta)+\mathcal{G}_{k}(\eta)\right)+\cdots,
$$

where

$$
\begin{aligned}
\mathcal{F}_{k}(\eta) & =\left(\int_{\eta_{0}}^{\eta} d \eta_{1} \frac{1}{v_{k}^{2}\left(\eta_{1}\right)} \int_{\eta_{0}}^{\eta_{1}} d \eta_{2} \frac{1}{u_{k}^{2}\left(\eta_{2}\right)}\right), \\
\mathcal{G}_{k}(\eta) & =\left(\int_{\eta_{0}}^{\eta} d \eta_{1} \frac{1}{u_{k}^{2}\left(\eta_{1}\right)} \int_{\eta_{0}}^{\eta_{1}} d \eta_{2} \frac{1}{v_{k}^{2}\left(\eta_{2}\right)}\right) .
\end{aligned}
$$

There some questions might arise about the entity of this state. We know that this state cannot be a vacuum state and should be written in terms of a superposition of excited states of $\phi$ and $\chi$. As a result, to gain such view one has to go to the Heisenberg language of the quantum field. In the next section, we shall try to obtain this goal and find a solution for (51). Note that the Eq. (49) is not the general entanglement solution for the system under study because it can not include all entangled states such as a state of form $\left|0_{\phi}, 1_{\chi}\right\rangle+\left|1_{\phi}, 0_{\chi}\right\rangle$.

\section{ENTANGLEMENT TRANSFORMATION}

In this section, we introduce a transformation like the Bogoliubov transformation which help us to reconstruct the previous entangled state in the Heisenberg picture. For simplicity, consider annihilation operator (28) for $\phi$ in the new form

$$
a_{k}=\mu_{k} \phi_{k}(\eta)+i \sigma_{k} \frac{\delta}{\delta \phi_{-k}}
$$

where

$$
\mu_{k} \equiv \frac{1}{i \sqrt{2}}\left[\left(a v_{k}^{\prime}(\eta)-a^{\prime} v_{k}(\eta)\right)\right]
$$

and

$$
\sigma_{k} \equiv \frac{1}{i \sqrt{2}} \frac{v_{k}}{a}(\eta)
$$

The similar annihilation operator for $\chi$

$$
b_{k}=\tilde{\mu}_{k} \chi_{k}(\eta)+i \tilde{\sigma}_{k} \frac{\delta}{\delta \chi_{-k}}
$$

where $\tilde{\mu}$ and $\tilde{\sigma}$ are similar to $\mu$ and $\sigma$ just with this different that $v_{k} \rightarrow u_{k}$ and $u_{k} \rightarrow v_{k}$. Note that we have absorbed $(2 \pi)^{3}$ factor just by redefinition of commutators (20) and (22). Now, we transform $a_{k}$ and $b_{k}$ to the new annihilation and creation operators as following

$$
\tilde{a}_{k}=\alpha_{k}(\eta) a_{k}+\beta^{*}(\eta)_{k} b_{-k}^{\dagger}
$$

$$
\tilde{b}_{k}=\alpha_{k}(\eta) b_{k}+\beta_{k}^{*}(\eta) a_{-k}^{\dagger}
$$

Here $\alpha_{k}$ and $\beta_{k}$ are like what we saw in Bogoliubov transformation with this difference that a Bogoliubov transformation is time-independent and so does not change the space of mode functions. This transformation just transforms a set of two independent mode functions into another set, but the above entangled transformation mixes modes of different fields and also can have timedependency. Unfortunately, Kanno [15] supposed that this kind of transformation as a time-independent transformation without any reason. Here we consider the general case and suppose this transformation can be time-dependent and finally describe why this cannot be independent of time. If we request that these new operators satisfy their own commutation relations like (22) then we get

$$
\left|\alpha_{k}(\eta)\right|^{2}-\left|\beta_{k}(\eta)\right|^{2}=1
$$

Consequently, one can find out that the new vacuum state $|\tilde{0}\rangle$ (which annihilated by $\tilde{a}_{k}$ and $\tilde{b}_{k}$ ) is

$$
|\tilde{0}\rangle=N(\eta) \exp \left(-\sum_{k} \frac{\lambda_{k}(\eta)}{2} a_{k}^{\dagger} a_{-k}^{\dagger}\right)|0\rangle
$$

where $\lambda_{k}=\frac{\beta_{k}^{*}}{\alpha_{k}}$. Now, let us find these states in Schrödinger picture. To this end, we consider the following relations

$$
\left\langle\phi_{k}, \chi_{k}\left|\tilde{a}_{k}\right| \tilde{0}\right\rangle=\left\langle\phi_{k}, \chi_{k}\left|\tilde{b}_{k}\right| \tilde{0}\right\rangle=0
$$

and use (58) and (61) to obtain

$$
\begin{aligned}
& \alpha_{k}\left[\mu_{k} \phi_{k}(\eta) \Psi[\phi, \chi]+i \sigma_{k} \frac{\delta \Psi[\phi, \chi]}{\delta \phi_{-k}}\right] \\
& +\beta_{k}^{*}\left[\tilde{\mu}_{k}^{*} \chi_{k}(\eta) \Psi[\phi, \chi]+i \tilde{\sigma}_{k}^{*} \frac{\delta \Psi[\phi, \chi]}{\delta \chi_{-k}}\right]=0
\end{aligned}
$$

and

$$
\begin{aligned}
& \alpha_{k}\left[\tilde{\mu}_{k} \chi_{k}(\eta) \Psi[\phi, \chi]+i \tilde{\sigma}_{k} \frac{\delta \Psi[\phi, \chi]}{\delta \chi_{-k}}\right] \\
& \quad+\beta_{k}^{*}\left[\mu_{k}^{*} \phi_{k}(\eta) \Psi[\phi, \chi]+i \sigma_{k}^{*} \frac{\delta \Psi[\phi, \chi]}{\delta \phi_{-k}}\right]=0 .
\end{aligned}
$$

These are just two simple functional differential equations which can be solved directly. The interesting point is that the solution has the form of (49) with the following kernels

$$
A_{k}=-i a^{2}\left(\frac{v_{k}^{\prime}}{v_{k}}-\frac{a^{\prime}}{a}\right)+\lambda_{k} C_{k} \frac{u_{k}^{*}}{v_{k}}
$$

and 


$$
A_{k}=-i a^{2}\left(\left(\frac{v_{k}^{\prime}}{v_{k}}\right)^{*}-\frac{a^{\prime}}{a}\right)+\lambda_{k}^{-1} C_{k} \frac{u_{k}}{v_{k}^{*}}
$$

and the same for $B_{k}$ would be obtain only by $v_{k} \rightarrow u_{k}$ and $u_{k} \rightarrow v_{k}$. These are two algebraic equations which have the following solutions

$$
C_{k}(\eta)=\frac{2 a^{2}(\eta)}{-\lambda_{k}(\eta) u_{k}^{*}(\eta) v_{k}^{*}(\eta)+\lambda_{k}^{-1}(\eta) u_{k}(\eta) v_{k}(\eta)}
$$

and

$$
\begin{aligned}
A_{k}(\eta)= & -i a^{2}\left(\frac{v_{k}^{\prime}}{v_{k}}(\eta)-\frac{a^{\prime}}{a}(\eta)\right) \\
& +\lambda_{k}(\eta) \frac{2 a^{2}(\eta)}{-\lambda_{k}(\eta) u_{k}^{*}(\eta) v_{k}^{*}(\eta)+\lambda_{k}^{-1}(\eta) u_{k}(\eta) v_{k}(\eta)} \frac{u_{k}^{*}}{v_{k}}(\eta) .
\end{aligned}
$$

If the reader looks at the Kanno's paper, he or she will find out that the Kanno's results for $A_{k}, B_{k}$, and $C_{k}$ is different from what we have derived here. This happens because Kanno did not do the field redefinition and also wrote a wrong condition for the Wronskian of mode functions. Now, we request that the new vacuum be a solution of the Schrödinger equation and with this consideration that we should equate these kernels with those we have found in (50) and (51). This helps us to find expressions for $f_{k}$ and $g_{k}$ which defined in (52) as following

$f_{k}=\rho_{k} v_{k}(\eta)\left[\exp \left(2 i \int_{\eta_{0}}^{\eta} \frac{u_{k}{ }^{*} \lambda_{k}}{-\lambda_{k} u^{*}{ }_{k}\left|v_{k}\right|^{2}+\lambda_{k}^{-1} u_{k} v_{k}^{2}}\right)\right]$

and

$g_{k}=\zeta_{k} u_{k}(\eta)\left[\exp \left(2 i \int_{\eta_{0}}^{\eta} \frac{v_{k}{ }^{*} \lambda_{k}}{-\lambda_{k} v^{*}{ }_{k}\left|u_{k}\right|^{2}+\lambda_{k}^{-1} v_{k} u_{k}^{2}}\right)\right]$

where $\rho_{k}$ and $\zeta_{k}$ are constants. We can see the way which we passed to finding an obvious description of the entangled state (by using of entanglement transformation), leads us to the above relations for nonlinear equations in (52). In the above relations, in order to do the integrals in the exponents we have to know the time-dependent form of $\lambda_{k}$. But what does happen if one supposes that $\lambda_{k}$ is timeindependent? Let us to look at it more precisely. Comparing Eq. (71) with Eq. (56) we find out that $\lambda_{k}$ should be a function of entanglement parameter $c_{k}$. In fact $c_{k}=0$ implies that $\lambda_{k}=0$. When $c_{k} \ll 1$ one can show that $\lambda_{k}=c_{k} / 2$. As a result, $\lambda_{k}$ is approximately constant and Eqs. (73), (74) are consistent with (54), (55). However when we go ahead and consider the next powers of $c_{k}$ we find out that $\lambda_{k}$ cannot be time-independent. If we expand $\lambda_{k}$ in terms of the entanglement parameter, one can show that

$$
\lambda_{k}(\eta)=c_{k} / 2-c_{k}^{3} N_{k}(\eta)+\cdots
$$

where

$$
N_{k}(\eta)=\frac{\mathcal{F}_{k}+\mathcal{G}_{k}}{2 a^{2}}(\eta)+\frac{1}{8} \frac{u_{k}^{*} v_{k}^{*}}{u_{k} v_{k}}(\eta)
$$

This implies that the entangled state defined by entanglement transformation will coincide with the $\mathrm{ABH}$ state if $\lambda_{k}$ is time-dependent. It is interesting to calculate the power spectrum in this picture. Kanno [15] has shown that the power spectrum of $\phi$ in Schrödinger picture is the same as the Heisenberg picture power spectrum only up to the second order of $\beta_{k}$. Kanno expands the Albertch et al.'s result (for power spectrum in the Schrödinger picture) with smallness assumption of $\beta_{k}$. She did not get to her exact form for power spectrum which she finds in the Heisenberg picture [15]. In contrast with Kanno's analysis, we find out in our analysis the two power spectra do coincide exactly without any approximation. In fact, with make using of (71) and (72) and after some tedious calculations one can show that

$$
\left\langle\tilde{0}\left|\phi_{-k} \phi_{k}\right| \tilde{0}\right\rangle=\frac{1}{2 a^{2}}\left|v_{k}(\eta)\right|^{2}\left(\frac{1+\left|\lambda_{k}\right|^{2}}{1-\left|\lambda_{k}\right|^{2}}\right)=\frac{1}{2} \frac{B_{k R}}{A_{k R} B_{k R}-C_{k R}^{2}},
$$

in which the subindex $R$ stands for the real part of each variable. One could see in this analysis, we are not only able to solve mode functions of entangled space in term of entanglement transformation parameter but also rederive the power spectrum which people have found in Schrödinger analysis. There just remains a point which we should clarify and that is by using Heisenberg picture, one can access other entangled states in a simple way. It is easy to see from (65) that the $\mathrm{ABH}$ state creates an entanglement between states of two fields with the same number of particles, i.e. $\left|0_{\phi}, 0_{\chi}\right\rangle,\left|1_{\phi}, 1_{\chi}\right\rangle, \ldots$ To make an entangled state in which states with different fields with a different number of particles are entangled with each other, it is better (and usual) to go the Heisenberg picture and by using of creation operators create an excited state of arbitrary number of particles and then mix the resultant state with momentum dependent coefficients. Consequently, without concern, the final entangled state is a solution for Schrödinger equation.

\section{CONCLUSION}

An important aspect of the quantum physics is the entanglement which can have the observational effect on cosmic structure formation which has originated from quantum fluctuations in the early universe [5]. The motivation to study the quantum entanglement is also given by the possibility that the EFT (effective field theory) that 
explains inflation may be rising from some more fundamental theory at the start of inflation. People do not have a detailed description of this process, so they just resort to a phenomenological framework that simply considers the EFT comes up with a slightly more general form of the wave function than Bunch-Davies. Another point of view is that we are embracing hints from the data that there may be a small breaking of rotational invariance in the state of the universe, and are considering a simple extension of BunchDavies that allows for this kind of breaking. This breaking can be studied in some different ways which one of them appears when one notes to this fact that the inflaton would most likely not appear alone, but accompanied by a many of other degrees of freedom (d.o.f.) (including scalar fields, tensor modes etc.), all of which would also be in some quantum state at the early time. Considering the entangled states of these fields with the inflaton and with themselves seems quite sensible. The entangled Gaussian state which is the next to simplest state to a Gaussian, provides evidence for rotational invariance breaking in the data. For example, the nontrivial transformations of the tensor perturbations under the rotation group (which leads to an entangled state) allow for the breaking of rotational invariance [10]. Such breaking is constrained by current data, but might still be large enough to explain some of the large-scale anomalies [18] found in the CMB temperature anisotropy maps. To study the entanglement in the early universe, first Albrecht et al. [10] have shown that the initial entangled state can have some features on the observable power spectrum in the early universe and then Kanno attempted to construct these initial entangled state by making use of a transformation which was similar to the Bogoliubov transformation [15]. Besides showing that the Kanno's calculation and analysis were not correct in some parts, in this article we have explored an entangled transformation (which is introduced Heisenberg picture) role on the Schrödinger field theory of the FLRW model. In accordance with this article analysis, we conclude with some remarks: (i) The solution of the entangled coefficient $A, B, C$ in the Schrödinger picture can be written in terms of the time-dependent entangled transformation parameter in the Heisenberg formalism. The entangled coefficient in (62) is not the Bogoliubov transformation since it mixes the mode functions of different fields and cannot be interpreted as a different observer physics like in the Bogoliubov transformation.

(ii) We should mention that an entangled state [see Eq. (49)] defined by an entanglement transformation, is not, in general, a solution of the Schrödinger equation and one has to use the Schrödinger equation to find parameter $\lambda_{k}$ of the entanglement transformation while a Bogoliubov transformation keeps us in space of solutions of the Schrödinger equation.

(iii) We calculated the relation between two vacuum states which their mode functions are transformed by the Bogoliubov transformation in the Schrödinger picture.

(iv) We have derived the entangled parameter $C_{k}$ in the Schrödinger picture in term of the entangled transformation parameter $\lambda_{k}$ in the Heisenberg picture. This formalism verifies that the entanglement transformation depends on the conformal time.

(v) The power spectrum of the inflaton field can be presented both in terms of the entangled parameter of Schrödinger picture and the entangled transformation coefficient in the Heisenberg one which has the same value.

(vi) It was discussed and shown that the entanglement has a more deep meaning which cannot be presented in the form of the (49) and in term of the entanglement transformation (62). We have presented an example that cannot describe the entanglement (49) and (62). Moreover, the entanglement was discussed in the Eq. (49) and (62) describe the entanglement between two fields and do not quantify the entanglement between the excited state of each field.
[1] P. A. R. Ade et al. (Planck Collaboration), Planck 2015 results. XIII. Cosmological parameters, Astron. Astrophys. 594, A13 (2016).

[2] A. Einstein, B. Podolsky, and N. Rosen, Can quantummechanical description of physical reality be considered complete?, Phys. Rev. 47, 777 (1935).

[3] R. Horodecki, P. Horodecki, M. Horodecki, and K. Horodecki, Quantum entanglement, Rev. Mod. Phys. 81, 865 (2009).

[4] J. Maldacena and G. L. Pimentel, Entanglement entropy in de Sitter space, J. High Energy Phys. 02 (2013) 038.

[5] J. Maldacena, A model with cosmological Bell inequalities, Fortschr. Phys. 64, 10 (2016); J. Gallicchio, A. S. Friedman, and D. I. Kaiser, Testing Bell's Inequality with Cosmic Photons: Closing the Setting-Independence Loophole, Phys. Rev. Lett. 112, 110405 (2014); E. Martin-Martinez and N.C. Menicucci, Entanglement in curved spacetimes and cosmology, Classical Quantum Gravity 31, 214001 (2014); S. Choudhury, S. Panda, and R. Singh, Bell violation in the sky, Eur. Phys. J. C 77, 60 (2017); J. Handsteiner, A. S. Friedman, D. Rauch, J. Gallicchio, B. Liu, H. Hosp, J. Kofler, D. Bricher, M. Fink, C. Leung, A. Mark, H. T. Nguyen, I. Sanders, F. Steinlechner, R. Ursin, S. Wengerowsky, A. H. Guth, D. I. Kaiser, T. Scheidl, and A. Zeilinger, Cosmic Bell Test: Measurement Settings from Milky Way Stars, Phys. 
Rev. Lett. 118, 060401 (2017); J. Martin and V. Vennin, Obstructions to Bell CMB experiments, Phys. Rev. D 96, 063501 (2017); C. Leung, A. Brown, H. Nguyen, A. S. Friedman, D. I. Kaiser, and J. Gallicchio, Astronomical random numbers for quantum foundations experiments, arXiv:1706.02276.

[6] C. Kiefer, F. Queisser, and A. A. Starobinsky, Cosmological constant from decoherence, Classical Quantum Gravity 28, 125022 (2011).

[7] S. Capozziello, O. Luongo, and S. Mancini, Cosmological dark energy effects from entanglement, Phys. Lett. A 377, 1061 (2013).

[8] S. Capozziello and O. Luongo, Dark energy from entanglement entropy, Int. J. Theor. Phys. 52, 2698 (2013).

[9] S. Mancini, R. Pierini, and M. M. Wilde, Preserving information from the beginning to the end of time in a RobertsonWalker spacetime, New J. Phys. 16, 123049 (2014).

[10] A. Albrecht, N. Bolis, and R. Holman, Cosmological consequences of initial state entanglement, J. High Energy Phys. 11 (2014) 093; N. Bolis, A. Albrecht, and R. Holman, Modifications to cosmological power spectra from scalartensor entanglement and their observational consequences, J. Cosmol. Astropart. Phys. 12 (2016) 011.

[11] D. Polarski and A. A. Starobinsky, Semiclassicality and decoherence of cosmological perturbations, Classical Quantum Gravity 13, 377 (1996); C. Kiefer, D. Polarski, and A. A. Starobinsky, Quantum to classical transition for fluctuations in the early universe, Int. J. Mod. Phys. D 07, 455 (1998); E. Nelson, Quantum decoherence during inflation from gravitational nonlinearities, J. Cosmol. Astropart. Phys. 03 (2016) 022; C. P. Burgess, R. Holman, G. Tasinato, and M. Williams, EFT beyond the horizon: Stochastic inflation and how primordial quantum fluctuations go classical, J. High Energy Phys. 03 (2015) 090.

[12] A. Rostami and J. T. Firouzjaee, Schrödinger field theory in curved spacetime: In-In formalism and three-point function for inflationary background, arXiv:1610.05038.
[13] J. Martin and V. Vennin, Quantum discord of cosmic inflation: Can we show that $\mathrm{CMB}$ anisotropies are of quantum-mechanical origin?, Phys. Rev. D 93, 023505 (2016); G. Len, G. R. Bengochea, and S. J. Landau, Quasi-matter bounce and inflation in the light of the CSL model, Eur. Phys. J. C 76, 407 (2016); S. Kanno, J. P. Shock, and J. Soda, Quantum discord in de Sitter space, Phys. Rev. D 94, 125014 (2016); P. Canate, P. Pearle, and D. Sudarsky, Continuous spontaneous localization wave function collapse model as a mechanism for the emergence of cosmological asymmetries in inflation, Phys. Rev. D 87, 104024 (2013); A. Rostami and J. T. Firouzjaee, Quantum decoherence from entanglement during inflation, arXiv: 1705.07703 .

[14] S. Kanno, M. Sasaki, and T. Tanaka, Vacuum state of the Dirac field in de Sitter space and entanglement entropy, J. High Energy Phys. 03 (2017) 068.

[15] S. Kanno, A note on initial state entanglement in inflationary cosmology, Europhys. Lett. 111, 60007 (2015).

[16] V. Mukhanov, and S. Winitzki, Introduction to Quantum Effects in Gravity (Cambridge University Press, Cambridge, England, 2007).

[17] The Bogoliubov transformation relates different mode functions bases. In a spacetime that has an appropriate timelike killing vector like the flat spacetime, there is a privileged mode function which the Bogoliubov transformation can transform different mode function to this mode function and vice versa. Note that the Bogoliubov transformation which is written here can be applied to both the flat FLRW and the de Sitter FLRW model. The basic point is that since the asymptotic behavior of the de Sitter spacetime is completely different from the flat spacetime, the Bogoliubov transformation for de Sitter spacetime basically is different from the flat spacetime and the asymptotic flatness cannot be defined for them.

[18] P. A. R. Ade et al. (Planck Collaboration), Planck 2015 results. XVI. Isotropy and statistics of the CMB, Astron. Astrophys. 594, A16 (2016). 